\title{
Investigation on Application of Support Vector Machine Algorithm in the Experimental Simulation System for Mechanism Motion Reliability
}

\author{
Zhang Tong, Li Sen, Qiao Jia-dong \\ School of Mechanical Engineering, Beijing Institute of Technology, Beijing, China

\section{Email address:} \\ zh_t11@263.net (Zhang Tong), liseninbit@163.com (Li Sen), 18810999192@163.com (Qiao Jia-dong)
}

\section{To cite this article:}

Zhang Tong, Li Sen, Qiao Jia-dong. Investigation on Application of Support Vector Machine Algorithm in the Experimental Simulation System for Mechanism Motion Reliability. Science Discovery. Vol. 5, No. 1, 2017, pp. 12-18. doi: 10.11648/j.sd.20170501.13

Received: October 31, 2016; Accepted: January 10, 2017; Published: March 15, 2017

\begin{abstract}
The complexity of the mechanism brings great difficulty to the calculation of the mechanism motion reliability, and the computer simulation algorithm based on Monte Carlo method needs a great number of simulation. The further-development of LMS Virtual. Lab was carried out, and the least square support vector machine algorithm was used to construct the response surface proxy model. The PSO-GA algorithm is used to optimize the parameters and make the least square support vector machine own a better performance. For the coupling of multiple failure modes, the multiple response surfaces are combined to obtain the reliability of the system. The reliability calculation based on support vector machine algorithm is carried out for the crank slider mechanism and the cam swing bar mechanism, and the feasibility and efficiency of the method are verified by the Monte Carlo method.
\end{abstract}

Keywords: Reliability, Simulation, Support Vector Machine, Response Surface Models

\section{支持向量机的算法在机构可靠性仿真系统中的应用研究}

张泮, 李森, 乔嘉栋

机械与车辆学院, 北京理工大学, 北京, 中国

\section{邮箱}

zh_t11@263.net (张制)，1iseninbit@163.com(李森)，18810999192@163.com（乔嘉栋）

摘要: 机构的复杂性给机构可靠性的计算带来很大的困难, 然而基于蒙特卡洛法的计算机仿真算法需要进行大量的模 拟。利用VB. NET对LMS Virtual. Lab进行二次开发, 并利用最小二乘支持向量机算法构建响应面代理模型，构建机构可 靠性仿真系统, 为使最小二乘支持向量机具有更好的性能, 利用PSO-GA算法进行参数的寻优。针对多失效模式耦合的 情况, 利用建立的多重响应面进行联合抽样以获得系统的可靠度。针对对心曲柄滑块机构、凸轮摆杆机构进行了基于 支持向量机算法的可靠度计算，并通过蒙特卡洛法进行验证，证明了该方法的可行性与高效性。

关键词：可靠性，仿真，支持向量机，响应面 


\section{1. 引言}

机构运动可靠性是衡量机构质量性能的重要指标之 一, 研究机构在规定条件和规定时间内完成规定功能的能 力。机构可靠性的研究内容涉及机构强度可靠性、机构运 动可靠性、机构系统可靠性等, 需要综合运用可靠性原理、 机构运动学、机构动力学、摩擦磨损原理等相关学科的相 关理论进行协同分析 [1]。

机构可靠性分析首先需要建立合理的可靠性模型, 由 于机构的制造误差、工作环境以及载荷、驱动系统的电、 液、力特性的变化, 都会使机构的输出特性与期望值产生 一定的误差, 且实际使用中的常存在碰撞、以及刚柔耦合 等因素, 导致可靠性模型难以建立, 引入新的影响因素后 会导致建模过程的工作量以及计算量急剧增加。在机构可 靠性的计算中, 一次二阶矩法、改进的一次二阶矩法、二 次二阶矩法等经典的可靠性算法受限于维数与显式的极 限状态方程, 然而由于现实中的机构可靠性受多方面因素 的影响, 且通常显式的极限状态方程通常难以获得, 经典 方法在复杂机构的分析中通常难以使用。由于机构失效模 式众多, 机构可靠性研究工作多针对于单一失效, 对于多 失效模式常采用串联模型或并联模型等进行简化计算, 无 法考虑失效模式间的耦合，导致对机构系统可靠性过于激 进或保守的估计。

基于蒙特卡洛法的计算机仿真算法是解决机构可靠 性问题的一种有效方法。纪玉杰等利用 $\mathrm{VC}++$ 、ADAMS以及 $\mathrm{CAD}$ 软件之间的相互协作开发了机构可靠性仿真系统 MARSS, 并利用此系统进行了某航炮可靠性模型的建模与 仿真 [2]。王慧等利用VB. NET对LMS Virtual. Lab进行二次 开发, 构建了可靠性仿真平台, 并对曲柄滑块机构、某舱 门锁机构进行了可靠性仿真, 对多目标进行参数化, 得到 了可靠度指标并进行了灵敏度的分析 [3]。程刚等编制基 于ADAMS 的参数化仿真驱动程序, 建立转管自动炮高速供 弹机构模型, 对多个拨弹轮初始定位角误差进行随机抽样 仿真并进行运动可靠性分析 [4]。但在实际使用中, 虽然 蒙特卡洛法可以得到较高的可靠性精度, 但必须要以巨大 的仿真量为代价, 对于涉及刚柔耦合、弹性变形等的复杂 机构, 会占用大量的时间以及计算机内存资源。本文提出 一种基于LMS Virtual. Lab的最小二乘支持向量机可靠度 算法, 通过VB. NET开发程序驱动LMS Virtual. Lab进行仿 真，利用支持向量机算法进行机构响应面的拟合，通过少 量的仿真计算就可计算出机构的可靠性指标, 大大减少了 仿真算法的计算量, 通过对于各失效模式对应的响应面进 行联合抽样, 可以获得机构的系统可靠性, 避免了传统串 联、并联模型的不足, 并对典型的对心曲柄滑块机构及凸 轮摆杆机构进行了可靠度的求解, 验证了该方法的可行性。

\section{2. 支持向量机响应面法}

响应面法 (Response Surface Method, RSM) 是通过一 系列确定性实验去拟合系统的输入与输出的函数关系的 方法。目前常用的响应面方法有多项式回归法、人工神经 网络法、支持向量机法等 [5], 多项式回归法常用二次多 项式进行拟合，但其对高阶非线性的曲面的反映能力不足，
在实际使用中发现, 通过增加多项式次数对拟合精度的提 高缓慢, 但待估计参数会呈指数增加, 神经网络法容易陷 入极小值, Kriging模型的参数估计计算复杂 [6] [7] [8]。

区别于传统的响应面法, 支持向量机响应面法利用支 持向量机回归拟合系统的输入与输出构造极限状态方程 的显式表达式。向量机（Support Vector Machine, SVM) 是90年代中期发展起来的基于统计学习理论的一种机器 学习方法, 通过寻求结构化风险最小来提高学习机泛化能 力, 实现经验风险和置信范围的最小化, 从而达到在统计 样本量较少的情况下, 亦能获得良好统计规律的目的。最 小二乘支持向量机 (Least Square Support Vector Machine, LSSVM) 由Suykens等提出, 用以解决非线性分类 以及回归问题。对于回归问题, 给定训练样本 $(\mathrm{x} 1, \mathrm{y} 1)$, $(\mathrm{x} 2, \mathrm{y} 2),(\mathrm{x} 3, \mathrm{y} 3), \cdots,(\mathrm{x} 1, \mathrm{y} 1)$, 建立回归函数 [9] [10] 即 回归超平面为:

$$
\mathrm{y}(\mathrm{x})=\omega^{T} \varphi(\mathrm{x})+\mathrm{b}
$$

$\varphi(\mathrm{x})$ 将样本空间非线性地映射到高维特征空间, $\omega$ 为 权重向量, 由结构风险最小化原则, 寻求参数 $\omega$ 和 $\mathrm{b}$, 使 目标函数 $\frac{1}{2}\left|\omega^{2}\right|$ 和误差项 $\xi_{\mathrm{i}}$ 最小, 采用等式约束, 将原始问 题转为求解凸二次规划问题:

$$
\begin{aligned}
\min \mathrm{J}(\mathrm{j}, \omega) & =\frac{1}{2}\|\omega\|^{2}+\frac{1}{2} \gamma \sum_{\mathrm{i}=1}^{1} \varepsilon_{i}^{2} \\
\mathrm{st}: \mathrm{y}_{i} & =\omega^{T} \varphi\left(x_{i}\right)+b+\varepsilon_{i}
\end{aligned}
$$

$\Gamma$ 为正则化系数, 引入拉格朗日乘子, 上述二次规划 问题转为:

$$
\mathrm{L}(\omega, b, \varepsilon, \alpha)=\mathrm{J}-\sum_{i=1}^{l} \alpha_{i}\left\{\omega^{T} \varphi\left(x_{i}\right)+b-y_{i}+\varepsilon_{i}\right\}
$$

$\alpha_{i}$ 为拉格朗日乘子, 由拉格朗日方程, 以下几个条件 必须满足: $\frac{\partial L}{\partial \omega}=0, \frac{\partial L}{\partial \mathrm{b}}=0, \frac{\partial L}{\partial \alpha_{i}}=0, \frac{\partial L}{\partial \varepsilon_{i}}=0$, 根据以上条 件, 则可得求解矩阵为:

$$
\left[\begin{array}{cc}
0 & 1_{v}^{T} \\
1_{v} & \Omega+\frac{1}{\gamma} I
\end{array}\right]\left(\begin{array}{l}
b \\
\alpha
\end{array}\right)=\left(\begin{array}{l}
0 \\
y
\end{array}\right)
$$

其中, $1_{v}=[1, \cdots, 1], I$ 为单位矩阵, $\Omega$ 为核矩阵:

$$
\Omega_{i j}=y_{i} y_{j} \varphi\left(x_{i}\right)^{T} \varphi\left(x_{j}\right)=y_{i} y_{j} K\left(x_{i}, x_{j}\right)
$$

则最小二乘支持向量机的回归函数为:

$$
\mathrm{y}(\mathrm{x})=\sum_{i=1}^{l} \alpha_{i} K\left(\mathrm{x}, x_{i}\right)+b
$$

式中, $\mathrm{K}\left(\mathrm{x}_{\mathrm{i}}, \mathrm{x}\right)$ 为核函数, 式 (4) 中的内积运算可由此 得到。支持向量机常用的核函数有: 线性核函数 $\mathrm{K}\left(\mathrm{x}_{\mathrm{i}}, \mathrm{x}\right)=\mathrm{x}_{\mathrm{i}} \cdot \mathrm{x}$; 多项式核 函数 $\mathrm{K}\left(\mathrm{x}_{\mathrm{i}}, \mathrm{x}\right)=\left[\left(\mathrm{x}_{\mathrm{i}} \cdot \mathrm{x}\right)+\right.$ $1]^{\mathrm{d}},(\mathrm{d} \in \mathrm{N})$; 高斯径向基核函数 $\mathrm{K}\left(\mathrm{x}_{\mathrm{i}}, \mathrm{x}\right)=\exp \left\{-\frac{\left\|\mathrm{x}_{\mathrm{i}}-\mathrm{x}\right\|^{2}}{2 \delta^{2}}\right\}$; $S$ 形核函数 $K\left(x_{i}, x\right)=\tanh \left(v\left(x_{i} \cdot x\right)+t\right)$ 。

区别于支持向量机 (Support Vector Machine, SVM), LSSVM把SVM的不等式约束变为等式约束, 使Lagrange乘子 
$\alpha$ 便于求解, 将 $Q P$ 问题转化为一个解线性方程组的问题, 具有较高的求解效率。

响应面法的内容主要包含实验设计、函数拟合, 此外 还应进行响应面的评定。由概率论与数理统计中的 $3 \delta$ 原则, 随机变量处于 $(\mu-3 \delta, \mu+3 \delta)$ 区间的概率为 0.9973 。基于 SVM的响应面法计算可靠度过程如下:

(1) 以 随 机变 量 的均 值 点 $\mathrm{X}^{(1)}=\left(x_{1 \mu}{ }^{(1)}, x_{2 \mu}{ }^{(1)}, \cdots, x_{\mathrm{n} \mu}{ }^{(1)}\right)$ 为初始迭代中心与 展开中心, $\mathrm{n}$ 为随机变量个数。

(2) 围绕展开中心进行抽样, 展开中心点取为拟合功能 函数的设计点 $\left(x_{1 D}^{(l)}, x_{2 D}^{(l)}, \cdots, x_{n D}^{(l)}\right)$ 与均值点按式 (7) 进行线性插值, 记为 $\left(x_{1}^{l}, x_{2}^{l}, \cdots, x_{n}^{l}\right)$, 展开抽样点为 $\left(x_{1}^{l}, x_{2}^{l} \pm f \delta, \cdots, x_{n}^{l}\right)$, 为验证响应面的精度, 在随机 变量的设计点附近随机抽取 5 组验证值, 通过调用 仿真程序进行模型的构建与仿真, 获取功能输出值。 $\mathrm{f}$ 为水平因子, 常取1 3, 为防止设计点落入迭代区
间之外, $\mathrm{f}$ 初始值选为 3 。为使在迭代过程中, 响应 面在设计点附近有更好的精确性, f值选为 1 。

$$
x_{i}^{l}=x_{i \mu}+\left(x_{i D}^{(l)}-x_{i \mu}\right) \frac{g(\mu)}{g(\mu)-g\left(x_{i D}^{(l)}\right)}
$$

（3）通过调用Matlab程序, 根据系统的训练样本, 利用 支持向量机算法拟合响应面, 并计算可靠度与设计 点。根据拟合的响应面对验证值进行预测, 计算预 测误差。

（4）判断设计点、可靠度指标与预测误差否均满足精度 要求。若条件满足, 则退出迭代, 若不满足, 重复 2 3步骤。由于支持向量机没有训练样本的限制, 为充分利用仿真数据, 将历次迭代的实验数据、验 证数据全部作为训练样本。

经过 $r$ 次循环得到系统的可靠度指标, 经分析, 共进 行 $2 n r+6 r$ 次仿真。

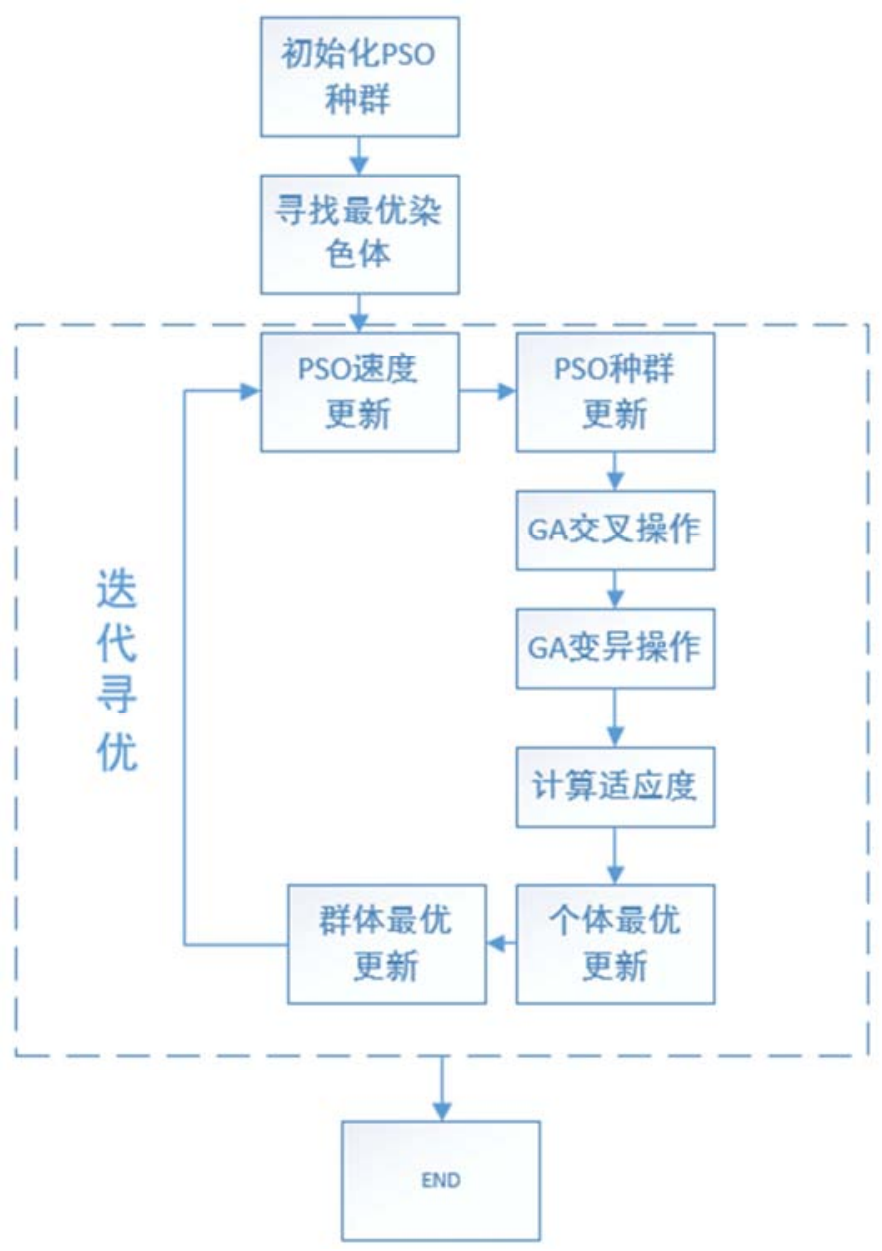

图1 $\mathrm{PS} 0 / \mathrm{GA}$ 寻优流程。

\section{3. $\mathrm{PS} 0 / \mathrm{GA}$ 参数寻优}

由于本文利用高斯径向基函数为核函数, 为使支持向 量回归机具有更好的性能, 需要对惩罚参数 $\mathrm{C}$ 和核函数参 数 $\delta$ 进行参数寻优。粒子群算法与遗传算法常被用来进行 LSSVM的参数寻优, 但两者均存在一定的缺陷。粒子群算
法对参数的选择具有较强的依赖性, 且无法保证获得全局 最优值, 可能陷入局部最优。遗传算法在后期求解效率低, 易早熟 [11] [12]。利用PSO/GA混合算法进行参数的寻优, 在支持向量机的训练中进行 $\mathrm{V}$ 折交叉验证, 以预测误差的 平均均方误差为适应度值, 寻优过程如图 1: 


\section{4. 可靠性仿真系统框架}

LMS Virtual. Lab与Nastran、CATIA具有良好的集成 性 [1], 利用LMS Virtua1. Lab作为机构运动仿真平台, Matlab编译数据统计与可靠性后处理模块, 利用VB. NET 开发软件界面, 实现对LMS Virtua1. Lab、CATIA、Nastran 的驱动以及Matlab的调用。可靠性仿真实验需要输入、仿 真、输出以及数据后处理等 4 方面的内容。可靠性仿真试 验的输入包含模型的建造、仿真环境的设置、离散化对象 的选取与随机数的抽取, 利用CATIA或LMS Virtual. Lab 进行虚拟样机的建造, 利用Nastra进行柔性体的分析; 为
实现仿真的自动运行, 将参数信息写入设计表, 通过程序 自动改变、更新模型的状态, 并进行循环仿真; 输出即为 根据研究对象获取仿真结果并进行输出与数据的整合, 在 仿真结果中选择待研究输出量进行保存, 减少无关因素对 数据库的占用; 在后处理模块中完成对仿真输入与输出信 息的分析, 利用设计表与输出参数作为训练样本训练响应 面, 并计算可靠度与设计点等参数。为验证所提响应面方 法的可行性，同时搭建基于蒙特卡洛法的可靠性仿真驱动 模块, 通过调用Matlab进行大量随机抽样以逼近真实的失 效概率。可靠性仿真实验系统框架如图2:

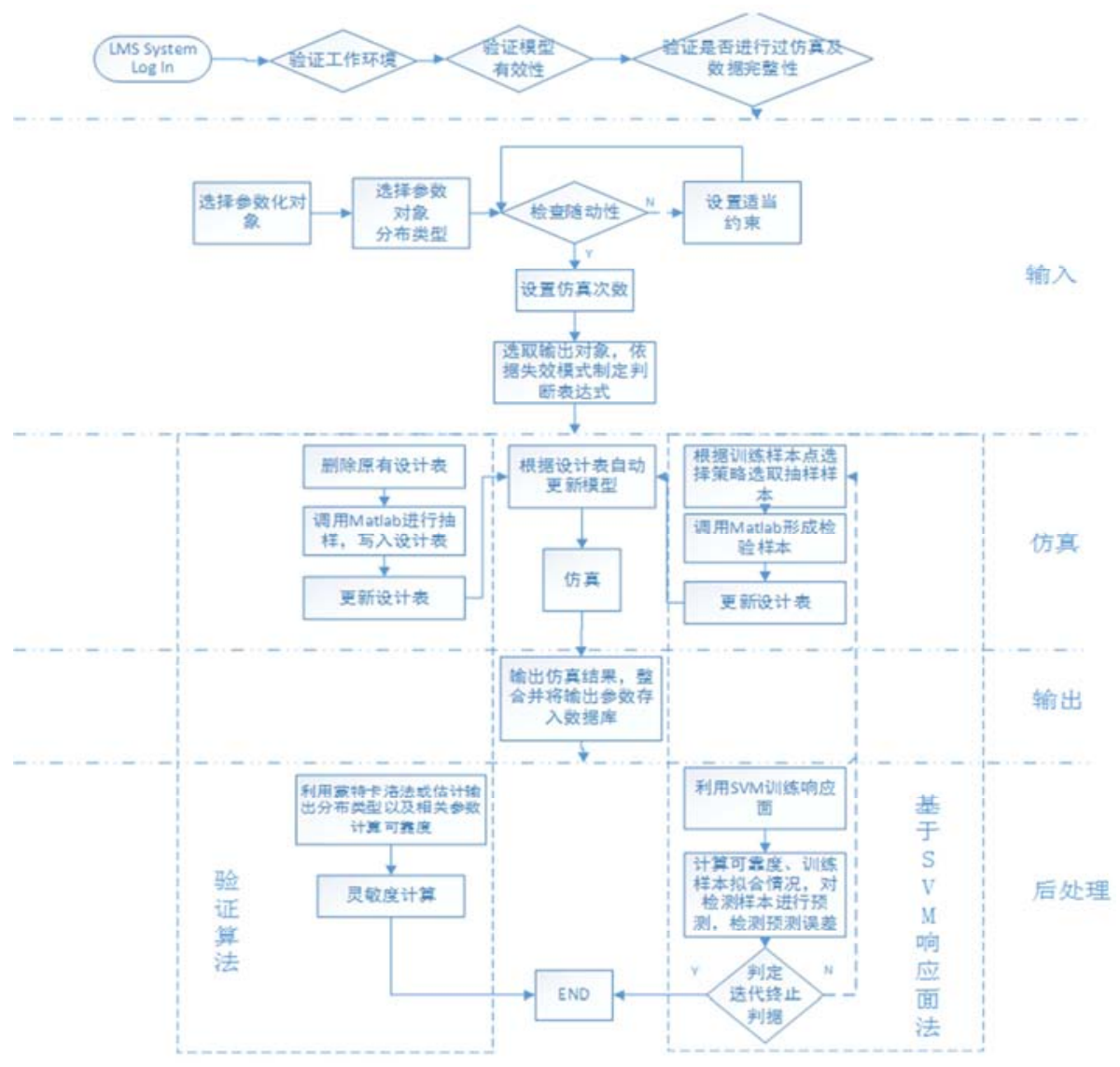

图2 可靠性仿真系统框架。

\section{5. 计算实例}

\section{1. 曲柄滑块机构}

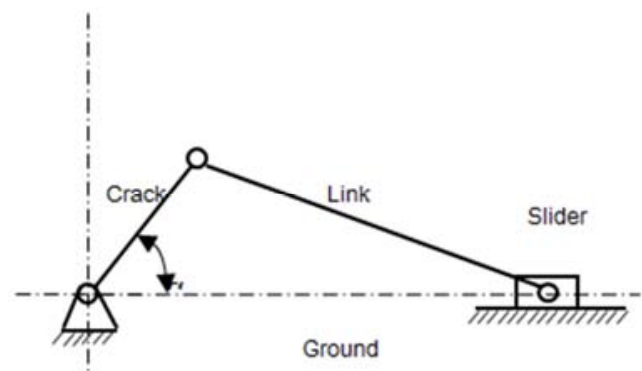

图3 曲柄滑块机构。
如图 3 所示对心曲柄滑块机构, 曲柄长度为 Crack $=200 \mathrm{~mm}$, 连杆长度为 Link $=400 \mathrm{~mm}$, 曲柄初始角度 $\alpha=0^{\circ}$, 转速恒定为 $1 \mathrm{rad} / \mathrm{s}$, 计算当 $\alpha=90^{\circ}$ 时, 考虑尺寸 精度误差, 滑块的位移、速度、加速度可靠性, 相关参数 如表1:

表1 机构参数表。

\begin{tabular}{lll}
\hline & 均值 $\boldsymbol{\mu}(\mathrm{mm})$ & 标准差 $\boldsymbol{O}$ \\
\hline Crack & 200 & 0.16667 \\
Link & 400 & 0.25 \\
Permissible Error (Displacement) & 0.4 & 0.1 \\
Permissible Error(Velocity) & 0.95 & 0.5 \\
Permissible Error (Acceleration) & 0.3 & 0.13 \\
\hline
\end{tabular}

建立图4所示虚拟样机，共包含4个部件：Ground、 Crack、Link、Slider。曲柄与地面之间采用旋转副连接, 
并施加驱动使角速度为 $1 \mathrm{rad} / \mathrm{s}$, 并将其置于Ground坐标系 的 $x$ 轴, 滑块与Ground添加平移运动副。在曲柄与连杆之 间、连杆与滑块之间添加旋转副。设置曲柄长、连杆长为 参数化变量, 滑块沿 $\mathrm{y}$ 轴的位移、速度、加速度 $(\mathrm{y} 、 \mathrm{yd}$ 、 $y d d)$ 为输出变量。

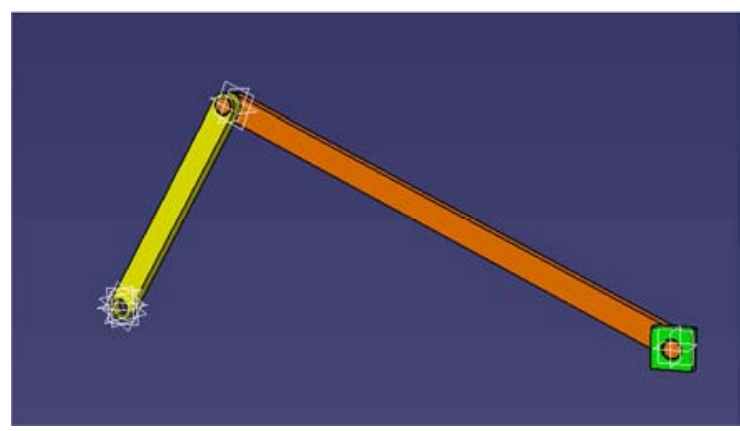

图4 对心曲柄滑块机构模型。

通过仿真计算, 当曲柄与连杆均为标准值时, 曲柄角 度为 $\alpha=90^{\circ}$ 时, 滑块位移为 $346.422 \mathrm{~mm}$, 速度为 $-0.2000 \mathrm{~mm} / \mathrm{s}$, 加速度为 $0.1154 \mathrm{~mm} / \mathrm{s}^{2}$ 。
通过基于SVM的响应面法的构建, 经过2次循环计算, 加速度可靠性已达到收玫值，共进行20次仿真，大大减少 了仿真时间。如图5所示, 经过响应面的建立, 对速度的 测试样本达到了良好的预测效果。

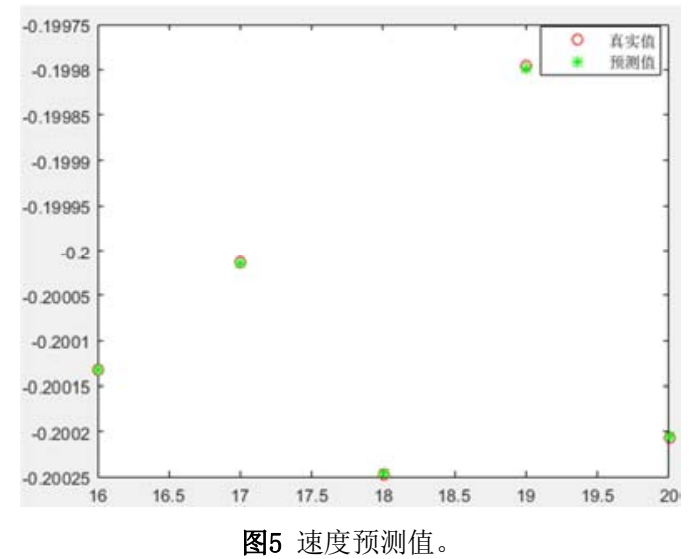

经过仿真计算，两种方法结果如下:

表2 结果对比。

\begin{tabular}{|c|c|c|c|c|c|c|c|c|c|}
\hline & \multicolumn{5}{|c|}{ Method1 } & \multicolumn{4}{|l|}{ Method2 } \\
\hline & 分布 & 拟合度 & 均值 & 方差 & 可靠度 & 循环次数 & 最大预测误差 & 可靠度 & 相对误差 \\
\hline 位移 & 正态 & 0.6663 & 346.408 & 0.0470 & 0.9379 & 4 & 0.0089 & 0.9269 & $1.17 \%$ \\
\hline 速度 & 正态 & 0.8371 & -0.2000 & 0.000159 & 0.9713 & 2 & 1. $2937 \mathrm{e}^{-06}$ & 0.9712 & $0.011 \%$ \\
\hline 加速度 & 正态 & 0.8535 & 0.11544 & 0.0002 & 0.9895 & 2 & 1. $7990 \mathrm{e}^{-06}$ & 0.9896 & $0.0101 \%$ \\
\hline
\end{tabular}

通过 200000 次联合抽样，得到位移可靠性为 0.9269 , 速度可靠性为 0.9712 , 加速度可靠性为 0.9896 , 若机构在 三种模式均不失效的情况下才能正常工作, 经统计, 机构 系统的可靠性为 0.9809 。若将系统视作串联系统, 系统可

\section{2. 凸轮摆杆机构}

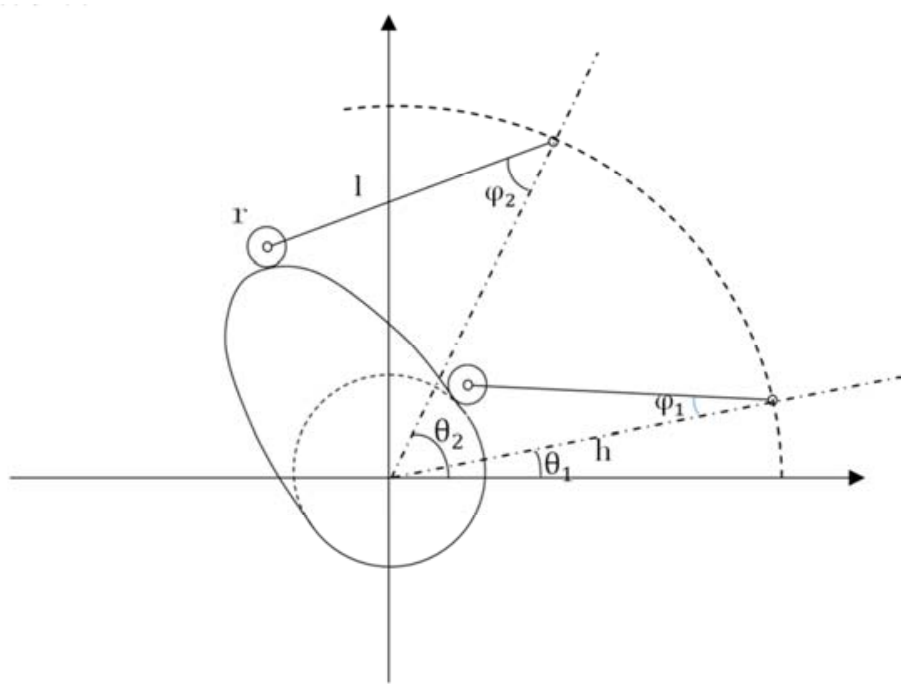

图6 凸轮摆杆机构。

如图6所示某盘形凸轮摆杆机构, 摆杆长 $\mathrm{l}=100 \mathrm{~mm}$, 滚子半径 $r=12 \mathrm{~mm}$, 摆杆铰链与凸轮铰链中心距离
靠性为 0.9808 。若机构在任一种模式不失效的情况下就能 正常工作，经统计，机构系统的可靠性为0. 999975, 若视 为并联系统，系统可靠性为 0.999978 。 
为 $\delta_{2}=120^{\circ}$, 近休止角为 $\delta_{3}=120^{\circ}$, 远休止角为 $0^{\circ}$, 凸 轮廓线符合 5 次多项式:

表3 凸轮廓线。

\begin{tabular}{lll}
\hline 角度 & 凸轮廓线方程 \\
\hline 推程 & $\delta_{1}=120^{\circ}$ & $\mathrm{s} 1=30 *\left(10\left(\frac{\theta}{\delta_{1}}\right)^{3}-15\left(\frac{\theta}{\delta_{1}}\right)^{4}+6\left(\frac{\theta}{\delta_{1}}\right)^{5}\right)$ \\
回程 & $\delta_{2}=120^{\circ}$ & $\mathrm{s} 2=30 *\left(1-10\left(\frac{\theta}{\delta_{2}}\right)^{3}+15\left(\frac{\theta}{\delta_{2}}\right)^{4}-6\left(\frac{\theta}{\delta_{2}}\right)^{5}\right)$ \\
近休止 & $\delta_{3}=120^{\circ}$ & $\mathrm{s} 2=30$
\end{tabular}

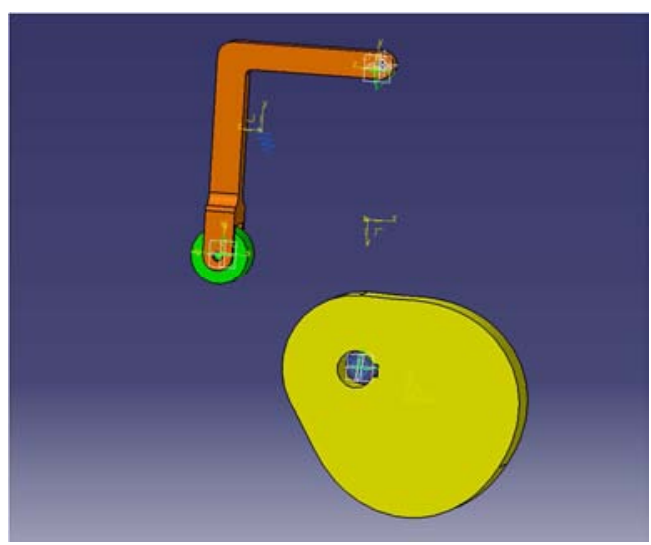

图7 凸轮摆杆机构模型。
凸轮按逆时针方向转动, 转动角度为 $\theta$, 转速 $\mathrm{n}=60 \mathrm{r} / \mathrm{min}$, 计算当 $\theta=90^{\circ}$ 时, 摆杆的角度与角速度 可靠性, 摆杆允许的角度误差特征值 $\left(\mu_{\mathrm{a}}, \sigma_{\mathrm{a}}\right)=$ $(0.3,0.05)$, 允许的角速度误差特征值 $\left(\mu_{\mathrm{ad}}, \sigma_{\mathrm{ad}}\right)=$ $(0.2,0.1)$ 。

建立图7所示虚拟样机, 摆杆与ground、摆杆与滚子、 凸轮与 ground之间均采用旋转副连接, 在摆杆上施加弹 簧力, 保证滚子不脱离凸轮表面。在凸轮与滚子之间添 加凸轮接触力, 模拟滚子与凸轮的接触与碰撞。对凸轮 旋转副施加驱动, 为使滚子与凸轮达到稳定良好的接触, 在仿真时设置凸轮转速为分段曲线, 使凸轮在滚子和摆 杆与凸轮达到稳定接触之前保持静止，之后以 $\mathrm{n}=60 \mathrm{r} / \mathrm{min}$ 的转速旋转。设置摆杆长度、滚子半径、摆 杆铰链与凸轮铰链中心距为参数化变量, 摆杆的角度、 角速度为输出变量。当参数化输入变量均为均值时, 经 过仿真计算, 凸轮转过 $90^{\circ}$ 时, 摆杆的摆角为 $26.9349^{\circ}$, 角速度为 $31.6558^{\circ} / \mathrm{s}$ 。

经过 1000 次仿真以及 $\mathrm{svm}$ 程序的计算，两种方法结果 如下:

表4 结果对比。

\begin{tabular}{|c|c|c|c|c|c|c|c|c|c|c|}
\hline & & Method1 & & & & & Method2 & & & \\
\hline & 分布 & 仿真次数 & 拟合度 & 均值 & 方差 & 可靠度 & 循环次数 & 最大预测误差 & 可靠度 & 相对误差 \\
\hline 角度 & 正态 & 1000 & 0.7456 & 26.9889 & 0.2671 & 0.9379 & 7 & 0.1586 & 0.9317 & $0.66 \%$ \\
\hline 角速度 & 正态 & 1000 & 0.9887 & 31.6527 & 0.0436 & 0.9687 & 5 & 0.0041 & 0.9765 & $0.98 \%$ \\
\hline
\end{tabular}

通过 200000 次联合抽样，得到角度可靠性为 0.9317 , 角速度可靠性为 0.9765 , 若机构在两种模式均不失效的情 况下才能正常工作, 经统计, 机构系统的可靠性为 0.9166 。 若将系统视作串联系统, 系统可靠性为0.9113。若机构在 任一种模式不失效的情况下就能正常工作, 经统计, 机构 系统的可靠性为 0.9934 , 若视为并联系统, 系统可靠性为 0.9985。

经过对比，支持向量机算法在极少的仿真次数下，取 得了较高的精度, 大大减小了仿真操作的工作量, 是一种 高效的可靠性分析方法。由于仿真次数较少，响应面的精 度以及可靠度计算方法的不同, 两种方法的结果存在着一 些微小的差距, 进一步提高效应面的精度与训练效率, 改 善可靠度算法, 将是本文今后的研究方向。

\section{6. 结论}

(1) 利用VB. NET对LMS Virtual. Lab进行二次开发，用 Matlab编制后处理模块，建立了机构可靠性仿真实 验系统。

（2）针对蒙特卡洛法需要大量仿真数据的不足，利用支 持向量机算法建立响应面并进行可靠度的计算。

（3）针对曲柄摇杆机构和凸轮摆杆机构进行了可靠度 计算, 验证了本文所提支持向量机算法的可行性。
经过少量的仿真计算便可得到较高精度的可靠度, 大大提高了效率。

（4）根据联合抽样的结果，传统的串联模型可能导致系 统可靠性的过于保守的估计, 并联模型的估计值可 能较为激进, 对于系统的可靠性反映可能是不准确 的。

(5) 由于弹性碰撞等因素的存在, 输出变量随机性较大, 造成响应面建立困难, 预测效果变差, 精度降低, 如何提高响应面的精度与适应性是亟待解决的问 题, 为进行复杂机构的可靠性计算提供更好的算法 支持。

\section{参考文献}

［1］王慧, 宋笔锋, 喻天翔. 基于多体仿真模型的运动机构可靠 性仿真试验系统研究 [J]. 宇航学 报, 2011, 32 (5) : 1005-1011。

[2] 纪玉杰. 机构动作可靠性仿真技术研究 [D]. 东北大学, 2006。

[3] 王慧, 喻天翔, 崔卫民, 等. 基于LMSVirtual. Lab平台的运动 机构可靠性分析和评估软件的开发 $[\mathrm{C}] / / 1 \mathrm{~ms}$ 中国用户大 会. 2010。 
[4] 程刚, 张相炎, 董志强, 等. 高速供弹机构运动可靠性仿真研 究 $[J]$. 兵工学报, 2011, $32(7): 801-804$ 。

[5] Li H, Lu Z. Efficient structural reliability assessment using support vector machine based response surface method $[\mathrm{C}] / / 2008$ Fourth International Conference on Natural Computation. IEEE, 2008, 2: 56-60.

[6] Samui P, Kothari D P. Utilization of a least square support vector machine (LSSVM) for slope stability analysis [J]. Scientia Iranica, 2011, 18 (1) : 53-58.

[7] $\mathrm{Xu} \mathrm{H}$, Chen G. An intelligent fault identification method of rolling bearings based on LSSVM optimized by improved PS0 [J]. Mechanical Systems \& Signal Processing, 2013, 35 (35): 167-175.

[8] Çomak E, Polat K, Güness S, et al. A new medical decision making system: Least square support vector machine (LSSVM) with Fuzzy Weighting Pre-processing [J]. Expert Systems with Applications, 2007, 32 (2) : 409-414.

[9] Ismail S, Shabri A, Samsudin R. A hybrid model of self-organizing maps (SOM) and least square support vector machine (LSSVM) for time-series forecasting [J]. Expert Systems with Applications, 2011, 38 (8) : 10574-10578.

[10] 张新锋, 赵彦. 基于最小二乘支持向量机的小样本威布尔可 靠性分析 [J]. 中国机械工程, 2012, 23 (16) : 1967-1971。

[11] Garg H. A hybrid PS0-GA algorithm for constrained optimization problems [J]. Applied Mathematics \& Computation, 2016, 274 (11) : 292-305.

[12] Chang J X, Bai T, Huang Q, et al. Optimization of Water Resources Utilization by PSO-GA [J]. Water Resources Management, 2013, 27 (10) : 3525-3540. 